\title{
High flow therapy versus hypertonic saline in bronchiolitis: randomised controlled trial
}

\author{
Mercedes Bueno Campaña, ${ }^{1}$ Jorge Olivares Ortiz, ${ }^{1}$ Cristina Notario Muñoz, ${ }^{1}$ \\ Marta Rupérez Lucas, ${ }^{1}$ Adelaida Fernández Rincón, ${ }^{2}$ Olga Patiño Hernández, ${ }^{1}$ \\ Cristina Calvo Rey ${ }^{2}$
}

- Additional material is published online only. To view please visit the journal online (http://dx.doi.org/10.1136/ archdischild-2013-305443).

${ }^{1}$ Department of Pediatrics and Neonatology, Hospital Universitario Fundación Alcorcón, Alcorcón, Comunidad de Madrid, Madrid, Spain ${ }^{2}$ Department of Pediatrics and Neonatology, Hospital Universitario Severo Ochoa, Leganes, Comunidad de Madrid, Spain

\section{Correspondence to} Dr Mercedes Bueno Campaña, Department of Pediatrics and Neonatology, Hospital Universitario Fundación Alcorcón, C/Budapest n¹ Alcorcón, Madrid 28922, Spain;

mbueno@fhalcorcon.es

Preliminary results presented as Oral Communication at the 61 st Congress of the Spanish Society of Paediatrics, June 2013, Granada, Spain.

Received 17 October 2013 Revised 9 January 2014 Accepted 17 January 2014 Published Online First 12 February 2014

\section{ABSTRACT}

Objective To demonstrate that heated humidified high-flow nasal cannula (HHHFNC) is superior to inhaled hypertonic saline solution (HSS) in improving respiratory distress in moderate bronchiolitis. In addition, it could improve comfort and reduce length of hospital stay (LOS) and admission to Paediatric Intensive Care Unit (PICU).

Design Randomised Clinical Trial from 1 October 2010 to 31 December 2012

Setting Two urban secondary (no PICU available) paediatric hospitalisation units.

Patients Hospitalised children aged up to 6 months with moderate acute bronchiolitis (Respiratory Distress Assessment Instrument, RDAI $\geq 4$ ).

Intervention Patients were randomised to HHHFNC or HSS. All of them received epinephrine as bronchodilator. Main outcomes Primary outcome was difference in mean Respiratory Assessment Change Score (RACS) between both groups measured in six previously defined consecutive moments. Secondary outcomes were difference in mean comfort scores in this period, LOS and rate of PICU admission.

Results Seventy-five previously healthy patients were enrolled. Mean age was 2.4 months $(95 \% \mathrm{Cl} 2.04$ to 2.76). 43 were allocated to HSS group and 32 in HHHFNC. Data of 1 patient were lost, and 8 changed group over the study period. Intention-to-treat principle was applied. There were no significant differences in mean RACS and mean comfort scores between groups at the evaluation points. Median LOS or PICU admission rate were similar in both groups. No adverse events were observed.

Conclusions HHHFNC was not superior to HSS in treatment of moderate acute bronchiolitis with respect to severity and comfort scores, LOS or PICU admission rate. Clinical Trial Registration ClinicalTrials.gov Identifier NCT01873144.

\section{INTRODUCTION}

Bronchiolitis is the most common lower respiratory tract infection in infants and represents an important cause of hospitalisation in this age group. It is estimated that $11-12 \%$ of all infants are affected in the first year of life, with 1-2\% requiring hospitalisation. ${ }^{1}$ Of those admitted, $10 \%$ of previously healthy infants and $36 \%$ of those with comorbidities will require Paediatric Intensive Care Unit (PICU), with 1\% of them dying. ${ }^{2}$ Pathological findings include airway wall swelling, increased mucus production which eventually leads to airway obstruction, atelectasis and impaired gas exchange. ${ }^{45}$ Standard treatment remains supportive and includes ensuring adequate oxygen

\section{What is already known on this topic}

- In bronchiolitis no therapy has conclusively shown to alter the course of the disease or its major outcomes.

- Standard treatment remains supportive.

- Considering pathological findings, any therapeutic modality which improves the clearance of airway secretions may be beneficial.

\section{What this study adds}

Heated humidified high-flow nasal cannula oxygen therapy is not superior to inhaled hypertonic saline solution in the treatment of infants diagnosed of moderate bronchiolitis.

exchange, fluid intake and feeding. Only in moderate severe bronchiolitis, trial with inhaled epinephrine is accepted. $^{67}$

Considering the pathological events, any therapeutic modality, like hypertonic saline solution (HSS), which improves the clearance of airway secretions may be beneficial. ${ }^{8}$ In a recent metanalysis, ${ }^{9}$ it was concluded that HSS should be considered an effective and safe treatment in mild-to-moderate acute viral bronchiolitis.

There is another promising therapeutic option, heated humidified high-flow nasal cannula (HHHFNC) oxygen therapy. It provides warmed and humidified gas with high flow, in theory improving work of breathing and comfort status. ${ }^{10-14}$ Research in HHHFNC has focused on preventing use of noninvasive and invasive ventilation in patients with acute bronchiolitis. ${ }^{11}$ 15-17 Though it has recently been gaining widespread popularity outside the PICU, ${ }^{18} 19$ there are no prospective, randomised trials which have demonstrated its utility in bronchiolitis.

We designed a clinical trial to demonstrate HHHFNC superiority versus HSS in improving respiratory distress in infants aged less or equal to 6 months hospitalised with moderate bronchiolitis. We also hypothesised that HHHFNC could improve comfort, shorten length of hospital stay (LOS) and reduce admission to PICU. 


\section{PATIENTS AND METHODS}

We conducted a controlled randomised clinical trial from 1 October 2010 to 31 December 2012 in two secondary paediatric hospitalisation units of Madrid (Spain). The participant hospitals attend a total population of 51269 children less than 14 years of age with no PICU available. The study was funded by Department of Health, Social Policy and Equality of Spain, Grant EC11-437 and approved by the Medical Ethics Committee of both centres.

Children aged 6 months or less presenting with moderate bronchiolitis (as defined by McConnochie ${ }^{20}$ ) and who met admission criteria were eligible for inclusion in the study. Moderate respiratory distress was defined by a Respiratory Distress Assessment Instrument (RDAI) score of four or greater (see online supplementary eTable 1). ${ }^{21}$ Exclusion criteria were: history of prematurity (gestational age less or equal 37 weeks), chronic lung disease, cystic fibrosis, congenital heart disease, neuromuscular disease, airway anomalies, immunodeficiency, and those requiring immediate intubation and ventilation. Informed consent was obtained from parents before enrolment.

The study was terminated at discharge or if, at any time, the clinical condition made the transfer to PICU necessary. Criteria for admission, discharge and transfer to PICU are shown in online supplementary eTable 2. Epidemiological and clinical data were recorded. Rapid test for respiratory syncytial virus and influenza in nasal swab was performed in all the patients.

Our nurses were trained in the clinical scoring system to ensure consistency and accuracy of scoring. There were three shifts of nurses every day.

\section{Study design}

Once included in the trial, participants received a nebulisation of $0.5 \mathrm{~mL} / \mathrm{kg}$ (maximum $3 \mathrm{~mL}$ ) of epinephrine $1 / 1000$ plus $2 \mathrm{~mL}$ of normal saline (NS) $(0.9 \%)$ if they had not received it previously. Then a computer-generated list was used $\mathrm{d}^{22}$ by investigators for simple allocation of the participants to two groups (ratio 1:1): (1) HSS Group: Nebulised epinephrine 1/1000 plus $2 \mathrm{~mL}$ of HS(3\%) every 4 h. (2) HHHFNC Group: HHHFNC with flow depending on weight (Tidal volume $\mathrm{x}$ respiratory rate $(\mathrm{RR}) \times 9)^{12}$ and nebulised epinephrine $1 / 1000$ plus $2 \mathrm{~mL}$ of NS $(0.9 \%)$ every $4 \mathrm{~h}$. Other treatments provided were intravenous fluids and supplement of oxygen adjusted to achieve oxygen saturation (SatO2) of 92-96\%. No other bronchodilators, antibiotic or steroid were used. In HSS Group, oxygen supplement was administrated by conventional nasal prongs, with flow not higher than $3 \mathrm{lpm}$. In HHHFNC Group flow was between 6 and $8 \mathrm{lpm}$. Physicians in charge were free to prescribe additional nebulisation or to change the patient's study group if deemed clinically necessary. RDAI score and RR were recorded by the nurse in charge, $30 \mathrm{~min}$ before and 60-90 min after concluding nebulisation, over three cycles, and afterwards every $8 \mathrm{~h}$ for $24 \mathrm{~h}$. Comfort score was recorded at admission and at the end of a nurse duty for $48 \mathrm{~h}$ after admission (see online supplementary eFigure 1).

\section{Study measurements and outcomes}

Respiratory Assessment Change Score (RACS) was considered as a measure of the efficacy of the assigned treatment. A RACS value of at least four has been previously defined as clinically relevant. The RACS is calculated as the difference between the RDAI score before and after treatment, plus a value of +1 for each $10 \%$ improvement (decrease) in the post-treatment RR or a value of -1 for each $10 \%$ worsening (increase) in RR. ${ }^{21} 23$
For comfort evaluation, a new scale designed by authors was used. It is based on four items: rest, feeding, alertness and facial expression (see online supplementary eTable 3 ). Its concordance was previously assessed in our Unit with 23 patients, obtaining a weighted $\kappa$ of 0.67 between parents and nurse (no published data).

Precision Flow (Vapotherm Inc. Stevensville, Maryland, US) and RT329 (Fisher and Paykel Healthcare, Auckland, New Zealand) were the dispositive used to administer HHHFNC depending on the availability. According to age, two different Fisher and Paykel nasal cannula were used in both devices, with different internare distance, and a maximum admitted flow of 6 and $8 \mathrm{lpm}$, respectively. Air leak around cannula in nares was allowed.

The variable for the primary outcome was the difference in mean RACS between groups at the evaluation points (RACSORACS5). For the first secondary outcome, it was the difference in mean comfort score over the monitoring period (Comfort1Comfort6). Other secondary outcome variables were LOS in days and admission to PICU (rate) in both groups.

\section{Statistical analysis}

For sample size estimate we considered a SD of three in our primary outcome. ${ }^{21}$ Seventy-five infants were required to detect a difference in mean RACS of two points, which was considered as clinically relevant by the authors ( $\alpha$ error of $0.05, \beta$ error of 0.2 and losses of 5\%). SPSS (V.19.0 for Windows) was used for statistical analysis. The intention-to-treat (ITT) principle was applied in all of our analyses. Per protocol analysis was subsequently performed. Means (SD) and median (IQR) are presented for continuous variables and rates for dichotomous ones. ANOVA with mixed model was used to study longitudinal data of RACS and comfort. This model was used to account for repeated measures within the same individual; as well this model allows working with unbalanced data, so we could include in the ITT analysis the subject with partial follow-up. ${ }^{24}$ The models included time as repeated factor, treatment as fixed effect and the interaction effect time*treatment. Post hoc pairwise comparisons of the least squared means for treatment at each point of time were contrasted with Bonferroni adjustment. PICU was compared between groups by $\chi^{2}$ test and LOS days were contrasted by Mann-Whitney U test; 95\% CIs are shown for the main results. The method of handling missing data was complete case analysis.

\section{RESULTS}

Seventy-five infants meeting inclusion criteria were enrolled (figure 1). Their mean age was 2.4 months (95\% CI 2.04 to 2.76). One patient from HSS Group was excluded from the analysis because all his data were lost. Of the 74 infants left, 42 (56.8\%) were enrolled in HSS Group and 32 (43.2\%) in HHHFNC Group. Both groups were similar at baseline (table 1). Sixty-six (89.2\%) infants stayed in the assigned group throughout the study. Eight patients were changed to HHHFNC Group; 79.7\% of patients had completed record, and only $5 \%$ of total data recorded was missing.

In mixed model for RACS, the interaction effect time-group was no statistically significant, $p=0.504$. Mean RACS (measure of efficacy) and mean comfort scale score were similar in both groups at the moments considered (table 2). Per protocol analysis showed significant differences in the RACS3 results $(1.88$; $95 \%$ CI 0.46 to 3.30$)$ and in Comfort4 results $(1.21 ; 95 \% \mathrm{CI}$ 0.03 to 2.38 ) favourable to HSS Group.

Both groups showed a parallel trend to improvement in their RDAI scores (measure of respiratory distress) during the 
Table 1 Baseline characteristics of the two groups

\begin{tabular}{lcc}
\hline & HSS ( $\mathbf{n = 4 2 )}$ & HHHFNC $(\mathbf{n = 3 2})$ \\
\hline Male (\%) & $22(53)$ & $11(35)$ \\
Mean age (months) (SD) & $2.65(1.70)$ & $1.95(1.27)$ \\
Mean baseline RDAl score (SD) & $6.67(1.98)$ & $7(1.81)$ \\
Mean RR (SD) & $51.1(11.9)$ & $49.9(12.8)$ \\
RSV positive (\%) & $31(74)$ & $23(72)$ \\
Mean previous illness duration (days) (SD) & $3.13(1.89)$ & $2.90(1.51)$ \\
\hline
\end{tabular}

HHHFNC, heated humidified high-flow nasal cannula; HSS, hypertonic saline solution HSS, hypertonic saline solution; RDAl, Respiratory Distress Assessment Instrument; RSV, respiratory syncytial virus.

follow-up (figure 2). In mixed model analysis there was no significant effect in RDAI $(p=0.24)$ or comfort $(p=0.7)$ scorings in the interaction time-group.

Fifty-eight patients did not suffer any complication. In five patients, the clinical status worsened and 10 needed transfer to PICU (table 1). One patient in HHHFNC Group asked for transfer to another centre not meeting criteria for PICU and was lost for complete follow-up. No adverse events were observed in any group. Fifty-five per cent $(75 / 145)$ of eligible patients were included in the study. The rate of transfer to PICU of patients not included was quite similar to the study group (13/70, 18.5\%).

\section{DISCUSSION}

Though our hypothesis was that HHHFNC could be superior to HSS, our results showed that in moderately ill patients, treatment with HHHFNC is no more effective than HSS as determined by a clinical scoring system (RADAI/RACS). Neither have we observed any differential benefit in comfort, LOS or rate of PICU transfer. This is the first published study that compared HHHFNC with HSS in the treatment of moderate bronchiolitis.

No therapy has conclusively shown to alter the course of bronchiolitis or its major outcomes. ${ }^{6} 7$ Epinephrine could improve comfort by reducing respiratory distress and a trial with this drug in infants suffering moderate-severe bronchiolitis is accepted in some guidelines. ${ }^{6725}$ Among all the published literature using HSS in the treatment of bronchiolitis, 11 trials were included in a recent meta-analysis ${ }^{9}$ which concluded that HSS reduces 1.15 day the mean LOS, compared to NS $(0.9 \%)$ and decrease significantly clinical severity score. It also conclude that despite the lack of strong evidence to recommend the routine use of HSS, its high safety profile, low cost and noninvasive administration make it a reasonable option for treating outpatient and inpatient children with bronchiolitis combined with a bronchodilator. ${ }^{7} 2627$

Based on the results obtained when used in neonatal setting, ${ }^{15}$ ${ }^{28-30}$ HHHFNC has been gaining considerable clinical support in the management of bronchiolitis. Retrospectives studies 11 17 31-33 have demonstrated a decrease in need for intubation parallel to the increase in use of HHHFNC in acute bronchiolitis. In prospective ones, a significant improvement in respiratory scale scores, oxygen saturation and comfort were also found using HHHFNC in patients with respiratory distress ${ }^{12}$ and bronchiolitis ${ }^{16}$ admitted to the PICU. In the last few years, HHHFNC has also become popular in hospitalisation wards as a method of delivering oxygen. It is easy to use and there may also be a perception that it offers a well-tolerated, non-invasive form of respiratory support. ${ }^{18} 19$ It is, however, a relatively expensive medical device with an on-going cost for consumables.

Though reduction in admission to PICU is the most important outcome in the treatment of bronchiolitis, to show this issue would have needed a larger sample. Given the incidence of the disease and considering the economical expense of bronchiolitis every season, other major indicators such as respiratory distress, comfort and LOS might be relevant when evaluating the most cost effective therapy. So far, there have not been well designed clinical trials published, whose main objective was to determine the effect of HHHFNC in these outcomes.

Our study has strengths we would like to point out. The first one is inclusion criteria. There is general consensus on the use

Table 2 Primary and secondary outcomes results

\begin{tabular}{|c|c|c|c|c|}
\hline & \multicolumn{2}{|l|}{ Mean (SE) } & \multirow[b]{2}{*}{ Mean difference* $(95 \% \mathrm{Cl})$} & \multirow[b]{2}{*}{ p Value* } \\
\hline & HSS ( $n=42)$ & HHHFNC ( $n=32)$ & & \\
\hline \multicolumn{5}{|l|}{ Primary outcome } \\
\hline RACSO & $1.24(0.5)$ & $0.91(0.56)$ & $0.33(-1.17$ to 1.84$)$ & 0.6 \\
\hline RACS1 & $0.95(0.48)$ & $0.38(0.55)$ & $0.57(-0.89$ to 2.03$)$ & 0.4 \\
\hline RACS2 & $0.36(0.39)$ & $0.39(0.46)$ & $0.07(-1.12$ to1.26) & 0.9 \\
\hline RACS3 & $0.19(0.49)$ & $-0.61(0.60)$ & $0.79(-0.75$ to 2.33$)$ & 0.3 \\
\hline RACS4 & $-0.18(0.54)$ & $1.04(0.70)$ & $-1.22(-2.99$ to 0.55$)$ & 0.2 \\
\hline RACS5 & $0.22(0.48)$ & $0.8(0.64)$ & $-0.58(-2.187$ to1.02) & 0.5 \\
\hline \multicolumn{5}{|l|}{ First secondary outcome } \\
\hline Comfort1 & $10.91(0.34)$ & $10.81(0.38)$ & $0.09(-0.93$ to 1.11$)$ & 0.9 \\
\hline Comfort2 & $11.60(0.29)$ & $11.35(0.34)$ & $0.25(-0.65$ to 1.12$)$ & 0.6 \\
\hline Comfort3 & $12.12(0.34)$ & $11.62(0.41)$ & $0.52(-0.54$ to 1.57$)$ & 0.3 \\
\hline Comfort4 & $12.45(0.38)$ & $11.87(0.46)$ & $0.58(-0.61$ to 1.77$)$ & 0.3 \\
\hline Comfort5 & $12.21(0.41)$ & $12.04(0.49)$ & $0.17(-1.09$ to 1.44$)$ & 0.8 \\
\hline Comfort6 & $12.77(0.38)$ & $12.95(0.46)$ & $-0.18(-1.38$ to 1.02$)$ & 0.8 \\
\hline \multicolumn{5}{|l|}{ Other secondary outcomes } \\
\hline LOS days median(IQR) & $4.5(3)$ & $5(4)$ & - & 0.8 \\
\hline PICU n (\%) & $5(11.9 \%)$ & $5(15.6 \%)$ & - & 0.5 \\
\hline
\end{tabular}


of the definition of McConnochie ${ }^{20}$ for bronchiolitis, based on age and clinical manifestations. Most of the studies comparing different therapies did not exclude previous wheezing ${ }^{16} 34$ or include patients older than 6 months ${ }^{16} \quad 23 \quad 34-36$ in whom the diagnosis of bronchiolitis may overlap with that of any other wheezing episode. We included only first episode of bronchiolitis in 6 months or younger in order to enrol patients with a more accurate diagnosis. Consequently, we think our results could reflect more suitably the response of bronchiolitis to the tested therapy. Second, the absence of a completely objective severity clinical scale makes it difficult to assess effectiveness. The RDAI/RACS score used in our study has demonstrated a high degree of inter-rater reliability ${ }^{21}$ and was applied by the nurses in charge (which can hampered the possible bias of subjectivity) resembling the everyday practice. Finally, we decided to design a comfort scale that took into account the assessment of parents and nurses (people closest to the patient) because comfort scales frequently used are based on response to sedation, tolerance in mechanical ventilation and level of pain, which are relevant outcomes of patients in intensive care, but not outside this setting.

Among limitations, the first one is that, though rate of transfer to PICU was similar in both groups and coincident with published data $^{3}$ the sample size was not calculated for such an infrequent event, so we cannot draw any strong conclusion from this result. Second, eight patients were changed from the original group assigned; all of them to HHHFNC Group. This fact broke randomisation. The decision of the physician in charge had priority, and probably, the change was justified due to worsening of their clinical status and the subjective impression in staff of a better outcome in patients treated with this therapy. We applied the ITT principle but per protocol analysis subsequently performed showed results only slightly favourable to HSS at one of evaluation points. These results reinforce those obtained by the ITT analysis. Third, there could be a potential seriousness bias since we excluded patients transferred directly to PICU. Albeit we only included in our study $50 \%$ of patients who were eligible, the rate of transfer to PICU of not included patients was quite similar to the study group. This could indicate that only logistical reasons, and not personal decisions based on severity criteria, are responsible for this event. And finally due to the techniques involved, the trial could not be blinded.

The variable named RACS measures the improvement in respiratory distress. In both treatment groups, mean RACS was less than 1.5 points, far from the value of at least four defined as a clinically relevant improvement due to a therapy. 2123 Though there was not a placebo group, the results obtained from the analysis of mix models suggest as well that the course of the illness was not modified by any of the treatment options tested.

\section{CONCLUSION}

HHHFNC was not superior to HSS in moderate bronchiolitis treatment with respect to severity scores, comfort or LOS. Neither has it reduced PICU admission rate respect to HSS but further studies with larger samples would be necessary to demonstrate this statement. Our study suggests that none of the therapies compared provide a real benefit to hospitalised infants less or equal to 6 months affected with moderate bronchiolitis.

Acknowledgements Elia Perez from Investigation Unit of the Hospital Universitario Fundación Alcorcon graciously provided assistance in the final statistical analysis. We thank all the nurses who assisted us with our work and, most of all, the families that participated in the study.

Contributors All authors contributed to the planning and writing of the manuscript. MBC performed the main part of statistical analysis and is the guarantor

Funding All phases of this study were supported by a grant of the Department of Health, Social Policy and Equality of Spain, Grant EC11-437.

Competing interests None.

Ethics approval Ethics Committee of both participant hospitals.

Provenance and peer review Not commissioned; externally peer reviewed.

\section{REFERENCES}

1 Meissner HC. Selected populations at increased risk from respiratory syncytial virus infection. Pediatr Infect Dis J 2003;22:S40,4; discussion S44-5.

2 Shay DK, Holman RC, Roosevelt GE, et al. Bronchiolitis-associated mortality and estimates of respiratory syncytial virus-associated deaths among US children, 19791997. J Infect Dis 2001;183:16-22.

3 Fitzgerald DA. Viral bronchiolitis for the clinician. J Paediatr Child Health 2011:47:160-6.

4 Smyth RL, Openshaw PJ. Bronchiolitis. Lancet 2006;368:312-22.

5 Bush A, Thomson AH. Acute bronchiolitis. BMJ 2007;335:1037-41.

6 American Academy of Pediatrics Subcommittee on Diagnosis and Management of Bronchiolitis. Diagnosis and management of bronchiolitis. Pediatrics 2006;118:1774-93.

7 Gonzalez de Dios J, Ochoa Sangrador C, Grupo de revision y panel de expertos de la Conferencia de Consenso del Proyecto aBREVIADo (BRonquiolitis-Estudio de Variabilidad, Idoneidad y ADecuacion). Consensus conference on acute bronchiolitis (I): methodology and recommendations. An Pediatr (Barc) 2010;72:221.e1-e33.

8 Mandelberg A, Amirav I. Hypertonic saline or high volume normal saline for viral bronchiolitis: mechanisms and rationale. Pediatr Pulmonol 2010;45:36-40.

9 Zhang L, Mendoza-Sassi RA, Wainwright C, et al. Nebulised hypertonic saline solution for acute bronchiolitis in infants. Cochrane Database Syst Rev 2013;7: CD006458.

10 Dysart K, Miller TL, Wolfson MR, et al. Research in high flow therapy: mechanisms of action. Respir Med 2009;103:1400-5.

11 Urbano J, del Castillo J, Lopez-Herce J, et al. High-flow oxygen therapy: pressure analysis in a pediatric airway model. Respir Care 2012;57:721-6.

12 Spentzas T, Minarik M, Patters AB, et al. Children with respiratory distress treated with high-flow nasal cannula. J Intensive Care Med 2009;24:323-8.

13 El-Khatib MF. High-flow nasal cannula oxygen therapy during hypoxemic respiratory failure. Respir Care 2012;57:1696-8.

14 Cuquemelle E, Pham T, Papon JF, et al. Heated and humidified high-flow oxygen therapy reduces discomfort during hypoxemic respiratory failure. Respir Care 2012;57:1571-7

15 Lampland AL, Plumm B, Meyers PA, et al. Observational study of humidified high-flow nasal cannula compared with nasal continuous positive airway pressure. $J$ Pediatr 2009:154:177-82.

16 Arora B, Mahajan P, Zidan MA, et al. Nasopharyngeal airway pressures in bronchiolitis patients treated with high-flow nasal cannula oxygen therapy. Pediatr Emerg Care 2012;28:1179-84

17 McKiernan C, Chua LC, Visintainer PF, et al. High flow nasal cannulae therapy in infants with bronchiolitis. J Pediatr 2010;156:634-8.

18 Hilliard TN, Archer N, Laura $\mathrm{H}$, et al. Pilot study of vapotherm oxygen delivery in moderately severe bronchiolitis. Arch Dis Child 2012;97:182-3.

19 Gonzalez Martinez F, Gonzalez Sanchez MI, Rodriguez Fernandez R. Clinical impact of introducing ventilation with high flow oxygen in the treatment of bronchiolitis in a paediatric ward. An Pediatr (Barc) 2013;78:210-15.

20 McConnochie KM. Bronchiolitis. What's in the name? Am J Dis Child 1983:137:11-13.

21 Lowell DI, Lister G, Von Koss $\mathrm{H}$, et al. Wheezing in infants: the response to epinephrine. Pediatrics 1987;79:939-45.

22 Macro !RNDI for SPSS Statistics. Extraction of N Random Integers from LN to HN (Exhaustive sampling) [computer program]. Bellaterra: Universitat Autònoma de Barcelona;'78. http://www.metodo.uab.cat/macros.htm (accessed 28 Apr 2013).

23 Grewal S, Ali S, McConnell DW, et al. A randomized trial of nebulized 3\% hypertonic saline with epinephrine in the treatment of acute bronchiolitis in the emergency department. Arch Pediatr Adolesc Med 2009;163:1007-12.

24 Cnaan A, Laird NM, Slasor P. Using the general linear mixed model to analyse unbalanced repeated measures and longitudinal data. Stat Med 1997:16:2349-80.

25 Evidence-based care guideline for management of first time episode bronchiolitis in infants less than 1 year of age. http://www.guideline.gov/content.aspx?id=34411 (accessed Sept 2013).

26 Da Dalt L, Bressan S, Martinolli F, et al. Treatment of bronchiolitis: state of the art. Early Hum Dev 2013:9(Suppl 1):S31-6. 
27 Nicolai A, Ferrara M, Schiavariello C, et al. Viral bronchiolitis in children: a common condition with few therapeutic options. Early Hum Dev 2013. http://dx.doi.org/10. 1016/j.earlhumdev.2013.07.016 (accessed 12 Oct 2013).

28 Holleman-Duray D, Kaupie D, Weiss MG. Heated humidified high-flow nasal cannula: use and a neonatal early extubation protocol. J Perinatol 2007;27:776-81.

29 Spence KL, Murphy D, Kilian C, et al. High-flow nasal cannula as a device to provide continuous positive airway pressure in infants. J Perinatol 2007;27:772-5.

30 Lee JH, Rehder KJ, Williford L, et al. Use of high flow nasal cannula in critically ill infants, children, and adults: a critical review of the literature. Intensive Care Med 2013:39:247-57.

31 Schibler A, Pham TM, Dunster KR, et al. Reduced intubation rates for infants after introduction of high-flow nasal prong oxygen delivery. Intensive Care Med 2011;37:847-52.
32 Onoro G, Perez Suarez E, Iglesias Bouzas Ml, et al. Severe bronchiolitis. Changes in epidemiology and respiratory support. An Pediatr (Barc) 2011;74:371-6.

33 Urbano Villaescusa J, Mencia Bartolome S, Cidoncha Escobar E, et al. Experience with high-flow nasal cannula oxygen therapy in children. An Pediatr (Barc) 2008:68:4-8.

34 Kuzik BA, Flavin MP, Kent $\mathrm{S}$, et al. Effect of inhaled hypertonic saline on hospital admission rate in children with viral bronchiolitis: a randomized trial. CJEM 2010;12:477-84

35 Mandelberg A, Tal G, Witzling M, et al. Nebulized 3\% hypertonic saline solution treatment in hospitalized infants with viral bronchiolitis. Chest 2003;123:481-7.

36 Al-Ansari K, Sakran M, Davidson BL, et al. Nebulized $5 \%$ or $3 \%$ hypertonic or $0.9 \%$ saline for treating acute bronchiolitis in infants. J Pediatr 2010;157:630-4. 